\title{
Ethnicity and Ethnic Identity in Context
}

\author{
Commentary on Gjerde \\ Lisa Kiang \\ Wake Forest University, Winston-Salem, N.C., USA
}

\author{
Key Words \\ Context · Developmental psychology · Ethnic identity · Ethnicity
}

As a developmental psychologist (DP) - the abbreviation refers to both practitioners and the discipline - who engages in ethnicity research, I read Gjerde's paper [this issue] with great interest and welcome the opportunity to provide my own evaluation and critique. Ultimately, Gjerde succeeds in what I sense is his broadest goal - to drive DPs to think deeply about the way ethnicity and ethnic identity have been conceptualized and investigated. Although Gjerde states that he does not necessarily recommend a "full-scale rebellion in which the field of DP would be radically transformed," while reading his arguments, I repeatedly found myself concerned about the poor, proverbial baby being mistakenly thrown out with the bathwater. If the conceptual water temperature is getting a little tepid, I can see the value in heating things up a bit. Gjerde conveys meaningful points that have the potential to influence the way we study ethnicity. However, while flaws and shortcomings of developmental research exist, I continue to see merit in some of the approaches that he challenges. I also argue that many DPs are already incorporating Gjerde's recommendations, which makes some of his criticisms seem unjustified. In this commentary, I highlight what I think are his most insightful points as well as offer my own evaluation with the consequent aim of providing a more balanced view of the state of ethnicity and ethnic identity research.

\section{What Gjerde's Views Offer (or, Freshening the Water, but Remembering Baby's Still There)}

Gjerde's critique centers on two major, and highly related, take-home points ethnicity is a social construction and is fluid and dynamic in nature. He also discusses additional recommendations such as for DPs to see the value in interdisciplin-

\begin{tabular}{ll}
\hline KARGER & ○ 2014 S. Karger AG, Basel \\
E-Mail karger@karger.com & $0018-716 X / 14 / 0574-0213 \$ 39.50 / 0$ \\
www.karger.com/hde &
\end{tabular}

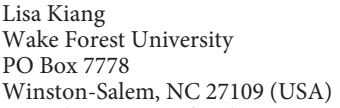


ary approaches, the need to consider intersectionality, and caution in using ethnic aggregates. Each of these points is highlighted here along with notable examples of recent work by DPs to illustrate how the field is currently addressing the criticisms that Gjerde brings to our attention.

\section{Ethnicity Is a Social Construction}

One prominent and recurring theme that Gjerde presents is that ethnicity is "embedded in social, historical, and political contexts." I agree with the importance of reminding DPs and others that ethnicity is a social construction and is, by nature, dynamic, flexible, and dependent upon groups, contexts, situations, political climates, social relationships, interactions, and more. Gjerde repeats this view in discussing the Multigroup Ethnic Identity Measure (MEIM) [Phinney, 1992], boundary work, primordialism, categories/groups, and ethnicity/culture. The need to study ethnicity in context is well-taken. Most broadly, Gjerde makes a strong case for researchers to look deeper into how ethnicity is shaped by historical and political influences. Few DPs, if any, would argue that development happens in a vacuum, and I agree that we could extend existing research and knowledge by directly recognizing the sociohistorical forces that affect ethnicity and related constructs.

In fact, several unmentioned, yet integral, frameworks can be drawn upon for inspiration. The Ethnic and Racial Identity in the 21st Century Study Group recently provided an integrated conceptualization in a special section for Child Development [Umana-Taylor et al., 2014]. Their review comprehensively defines ethnic and racial identity as multifaceted and formed through dynamic processes, situating its development within specific sociohistorical contexts. Entrenched in the articles of this special section are further discussions of the impact of the social environment, methodological strategies, and issues related to traditional self-report measures of ethnicity and ethnic identity as well as more novel assessment techniques [Schwartz, Syed, Yip, Knight, Umana-Taylor, Rivas-Drake, \& Lee, 2014]. García Coll, Crnic, Lamberty, Wasik, Jenkins, García, and McAdoo's [1996] integrative model dates back almost two decades and also highlights the role of sociocultural influences in the lives of ethnic minority children, recognizing cultural legacies, economic and political histories, acculturation and migration, and other contextual demands. Notably, this model also highlights social stratification, power, and social position variables, which Gjerde later discusses as another limitation of the field. Another conceptual model put forth by Mistry and Wu [2010] similarly focuses on community, familial, and individual factors in development, and treats cultural navigation as firmly situated in social contexts and interactions. The Multidimensional Model of Racial Identity (MMRI) is yet another framework that emphasizes the unique historical legacies and contemporary societal interactions that affect identity formation [Sellers, Smith, Shelton, Rowley, \& Chavous, 1998]. As a final example, the Society for Research in Child Development (SRCD) Asian Caucus recently developed a series of papers that note the need for increased awareness of how developmental processes, including ethnicity and ethnic identity, are influenced by both historical and contemporary contexts [Tseng \& Kiang, 2014]. Hence, my sense is that, like Gjerde, many others in the field are similarly frustrated by the limited attention to contextual factors in development, and they are making and continuing to make strides toward more deliberate investigations of such influences. 
Another layer of contextual complexity involves variation at a more local or regional level. One of Gjerde's criticisms is that DPs are not reaching out to certain geographic contexts, with undocumented farmworkers in the San Joaquin valley given as one example. Indeed, the bulk of American ethnicity research has centered on large, metropolitan communities rather than emerging immigrant areas that have not traditionally hosted newcomers and are only just experiencing exponential growth in their immigrant populations. Clearly, the developmental context of an urban, ethnically rich area such as Los Angeles is qualitatively different from, for instance, new settlement areas in rural California, small towns in the Midwest, or Western North Carolina. While I value Gjerde's call to broaden our research scope, I would be remiss not to acknowledge that many have been actively targeting such neglected populations and, in doing so, are better uncovering how context functions in development. The following are just a few prime examples: research on understudied Mexican immigrant children in predominantly White communities [Brown \& Chu, 2012], migrant farmworkers in emerging immigrant areas [Grzywacz, Quandt, Chen, Isom, Kiang, Vallejos, \& Arcury, 2010; Kiang, Grzywacz, Marin, Arcury, \& Quandt, 2010], comparative studies of immigrant youth residing in Los Angeles and emerging immigrant communities [Kiang, Perreira, \& Fuligni, 2011; Perreira, Fuligni, \& Potochnick, 2010], and research focusing exclusively on adolescents in new immigrant destinations [Gonzalez, Stein, Shannonhouse, \& Prinstein, 2012; Kiang, Supple, Stein, \& Gonzalez, 2012; Perreira, Chapman, \& Stein, 2006]. Ethnicity is not "context-free," and the growing literature reflects this important point.

\section{Ethnic Identity Is Fluid and Dynamic}

A closely related issue is that ethnic identities are "constantly 'in the making' they are emergent and not set in stone." Again, I doubt that many DPs, or any, would argue that ethnic identity is constant across age or context. Erikson [1968] himself viewed identity as evolving across time and situations. Although some measures implicitly suggest that ethnic identity can be captured through single-survey accounts, emerging approaches have more deliberately explored its fluidity. While Gjerde acknowledges some of these novel approaches, I think these measurement efforts deserve a little more elaboration.

Inspired by a social identity perspective, Sellers et al.'s [1998] MMRI defines identity as being comprised of multiple dimensions. Salience is one, which explicitly recognizes identity's situational fluctuation. Although the MMRI has been measured using a rather traditional self-report format, Yip and Fuligni [2002] assessed ethnic salience through the repeated measures of a micro-longitudinal daily diary approach and found that adolescents felt more "Chinese" on days in which they participated in ethnic-related events. Ethnic salience has also been measured even more experientially through palm pilots and cellphones [Yip, 2005, 2014]. Some of my work documenting relationship-specific variability in ethnic identity, as measured through adaptions of the MEIM, also speaks to Gjerde's argument that ethnicity is a "facet of a social relationship" and not "an attribute of a person or a cultural group" [Kiang \& Fuligni, 2009; Kiang, Harter, \& Whitesell, 2007]. We have also found support for similar relational variations in adolescent ethnic labeling 
preferences, both in terms of self-chosen labels [Kiang \& Johnson, 2013], as well as in discrepancies between self-chosen labels and those ascribed by others [Kiang \& Luu, 2013], the latter of which illustrates Gjerde's suggestion that ethnicity is, in part, imposed or directly influenced by social interactions. Person-centered approaches also provide insight into the dynamic nature of ethnic identity without limiting its potential change in one linear direction. For instance, using multiwave assessments of the MEIM, we have found that ethnic identity appears stable over time, which is perhaps consistent with Gjerde's criticisms around DPs who view identity as static [Kiang, Witkow, Baldelomar, \& Fuligni, 2010]. Yet, with an intraindividual approach, we correlated individual change in identity subscales with changes in other key variables (e.g., family cohesion, ethnic centrality), thereby using traditional self-report scales in a flexible way. Taken together, I again emphasize that many DPs are essentially doing the kind of work that Gjerde recommends, and there are innovative efforts to capture the variable and fluid nature of ethnicity and identity processes in development.

\section{Utility of Interdisciplinary Work}

Gjerde calls for DPs to integrate interdisciplinary research perspectives, and I agree that the field would benefit from thinning and flexing its boundaries. Barth's [1969] social anthropological perspective appears to be a highly useful framework that can be applied to the study of ethnicity and ethnic identity. Admittedly, this DP is guilty of not "reading outside the box" and thus appreciated learning about this relevant view. However, even though these early conceptualizations were not readily accessible to me does not mean that I was not already incorporating them in my work. I think that the same can be true for many of our colleagues. Indeed, I was a little uncomfortable with Gjerde's assertion that DPs need to take ethnography and field work more seriously. My contention is that I do not see much indication that DPs downgrade or dismiss these approaches. According to its mission statement, "The SRCD promotes interdisciplinary research on infant, child, and adolescent development in diverse contexts ...." The Society for Research on Adolescence (SRA) [2010] similarly states that they share a set of values that include "diversity in scholarship, including the study of diverse populations; disciplinary perspectives; and methodological approaches." Although not all DPs engage in interdisciplinary research, it is important to state that this is at least something many do value and perhaps strive toward.

\section{Importance of Intersectionality}

Rooted in Gjerde's discussion of intersectionality are several key points. One is the idea that ethnicity is just one of many possible influences in individuals' lives. Certainly, there is utility in examining multiple forms of identity and recognizing simultaneous and interactive associations between ethnicity, gender, sexuality, religion, etc. [Williams, Tolan, Durkee, Francois, \& Anderson, 2012]. However, simply adding more variables might be dangerous in that every new factor incorporated might be similarly prone to reification. Further, we cannot possibly incorporate $\mathrm{ev}$ - 
erything, so it is important not to disregard research efforts with a singular focus on, for instance, ethnicity. Another implication is the presumption that ethnicity is even important to begin with. While I value a holistic view and the recommendation to keep our assumptions in check, I disagree with the suggestion that DPs automatically assume that ethnicity is salient and overestimate its importance. The very reason why many DPs even measure ethnicity is because they (we) believe that there are individual differences in just how salient it is. Gjerde's later discussion of primordialism also touches on this issue. That is, do DPs mistakenly assume that ethnicity is a "real" phenomenon? One useful framework along these lines can be borrowed from cultural anthropology whereby "folk models" can be used to understand views of ethnicity as "essentialist" (e.g., innate, in the blood, brain) or "social constructivist" [Mahalingam, 2007]. As Gjerde suggests, the idea of examining whether individuals know, a priori, what their ethnicity is, or have confidence in that assessment, is an intriguing one. In many ways, this question equates to the very process of identity development, whereby individuals determine where they belong in terms of the groups and categories that exist.

\section{Problem with Aggregates}

I share Gjerde's apprehension over the use of ethnic aggregates and agree that we should consider how we view and ultimately categorize ethnicity. Recent trends toward reporting on disaggregated ethnic data corroborate this importance. For instance, the Asian American Federation [2014] recently highlighted the immense disparities among Asians; while many groups (e.g., Chinese, Korean, Indian) are relatively well-adjusted, some (e.g., Bhutanese, Hmong, Laotian) struggle on a number of different levels. Other theoretical and empirical work similarly touts the potential limitations of using broad, panethnic categories in research [Guarnaccia, Pincay, Alegría, Shrout, Lewis-Fernández, \& Canino, 2007; Supple, Graves, Daniel, Kiang, $\mathrm{Su}, \&$ Cavanaugh, 2013]. However, it is important to observe that, in some circumstances, a panethnic or aggregate grouping might actually be quite appropriate to the research questions and conceptualizations of a particular study. For instance, a panethnic "Asian American" classification is often used due to issues of statistical power; there are simply too few individuals who comprise a specific Asian group to reveal meaningful patterns or comparisons [Yoshikawa, Mistry, \& Wang, 2014]. As research continues to evolve, more attention should be directed towards our use and potential misuse of aggregates, which do need to be made at one point or another unless we engage in case studies. At best, these decisions should be driven by research questions. I hesitate to agree that aggregates should be uniformly criticized because, in some situations, a panethnic classification might make the most conceptual and practical sense.

\section{The Water Seems Fine (or, Disputes over the Applicability of Gjerde's Views)}

Related to the idea that ethnicity is a social construction, Gjerde distinguishes between ethnic categories and groups. The theme is that categories are broader and somewhat imposed by others, whereas groups reflect an intrapersonal sense of inter- 
dependence among group members themselves, presumably without this outside influence. Although I agree that the construct of ethnicity has become racialized, politicized, and privy to the hegemonic influences of others, it is not clear how this distinction is useful or even possible to empirically address other than simply recognizing that ethnicity, again, is shaped by others. I appreciate the warning that some DPs might define ethnicity too narrowly, but how we can use this distinction to better inform our research is uncertain. This point seems to boil down to basic differences in jargon.

I also questioned the utility of distinguishing between ethnic and cultural groups. This issue seems directly relevant to Barth's [1969] critical points of view in the sense that ethnicity, but not culture, is the result of social interactions. However, I wonder whether one can ever engage in social interactions that are truly context-free and that disregard, implicitly or explicitly, the "cultural stuff." What would this look like, practically speaking? Again, I respect the foundational message that ethnicity is a social construction, and outlining the differences and similarities between ethnicity and culture is engaging and thought-provoking, but I hesitate in seeing how making this distinction would move us beyond jargon and propel the field forward.

\section{In Support of the MEIM}

Although, like all self-report measures, the MEIM is not perfect, I do not think that all of Gjerde's criticisms are fair. For instance, the measure itself arguably does assess ethnic identity unilaterally, or, perhaps, attempts to capture its dynamism and dimensional nature in an ultimately static way. However, it is important to reiterate that Phinney [2003] has staunchly defined ethnic identity as "a dynamic, multidimensional construct that refers to one's identity or sense of self as a member of an ethnic group" (p. 63). Although questions circulate around its factorial structure [Lee \& Yoo, 2004; Phinney \& Ong, 2007; Roberts, Phinney, Masse, Chen, Roberts, \& Romero, 1999], I realize and agree with many researchers' motives for theoretically retaining the multidimensional distinctiveness of certain subscales. In addition, I appreciate the MEIM's Eriksonian origins in attempting to provide a window into identity processes. Although its standard use might imply constancy across age and context, I doubt that all or even many DPs assume that this sense of constancy is absolute. I view any self-report survey, as well as one-time narrative assessments for that matter, as a single snapshot of a respondent's life or current feelings, not as representative of something assumed to be constantly important or stable.

Another unfair criticism is the assertion that the MEIM uses pre-defined panethnic labels (e.g., Hispanic, Latino/a). Although Gjerde's warning against this rigid use of categories is worthy, it is vital to recall that the MEIM was initially created so that people could define ethnicity for themselves. Panethnic examples are given in the original instructions, as are clear statements on the many ethnic options available. The ability to self-define ethnicity is, to me, a great strength, and uses an individualized approach that Gjerde actually recommends. Granted, we could be more cognizant of how this or any other measure is used (e.g., how ethnicity is treated as a variable, whether we make biased assumptions across ethnic groups/ categories), but I do not think that the measure itself is fatally flawed in the ways that Gjerde suggests. 


\section{In Light of "What Needs to Be Done", What Can We Really Do?}

In light of how DPs' efforts to study ethnicity have presumably fallen short, I was eager to see Gjerde's recommendations to move the field forward. He discusses several ideas (e.g., consciousness model, person-centered frameworks), but most notably stresses the value of narratives. I certainly see merit in narratives, but I am not convinced that they are any better than self-report measures already in use. Even with narratives, researchers must construct aggregates based on responses and potentially reify in the process. Narratives do not automatically take into account contextual fluidity, short of conducting case studies where constant narrations are made while engaging in the "minutiae of everyday encounters". This singular recommendation also ignores the importance of using multiple methods to provide converging evidence for theories and hypotheses. Revisiting primordialism, using narratives to allow ethnicity issues to emerge spontaneously is an appealing technique. Yet, must something spontaneously come up for it to be deemed important? Cannot this argument be made for just about anything? For example, in everyday conversation, people might not spontaneously bring up issues of self-esteem or mental health, but does this mean that these constructs are not important to overall development and that we should not try to measure them directly? Hence, while narratives are valuable, we should be careful not to forsake other assessment tools, even the more traditional self-report surveys.

\section{Concluding Remarks}

In summary, Gjerde's evaluation has provided useful fodder for my own approach, and I suspect that many DPs interested in ethnicity will find his points pertinent and constructive. I appreciate most his reminder to treat ethnicity and ethnic identity as social constructions, highly malleable and context-dependent. There is value in interdisciplinary thinking and research, as well as deliberately considering the intersectionality between ethnicity and other factors such as gender, religion, sexuality, or social class. However, while there is always good reason to evaluate the temperature and freshness of our bathwater, we need to make sure that we retain the baby in the process. Moreover, after critical evaluation, we might even realize that the water is not as dirty as it seems.

\section{Acknowledgements}

I am grateful for the opportunity to respond to Per F. Gjerde's evaluation and critique and for Melissa R. Witkow, who provided feedback on an earlier draft of this commentary.

\section{References}

Asian American Federation (2014). The state of Asian American children. New York, NY: Asian American Federation. Retrieved from http://www.aafny.org/doc/AAF_StateofAsianAmericanChildren.pdf. Barth, F. (1969). Ethnic groups and boundaries: The social organization of culture difference. Oslo, Norway: Norwegian University Press. Reprinted in 1998 by Waveland Press in Long Grove, IL. 
Brown, C.S., \& Chu, H. (2012). Discrimination, ethnic identity, and academic outcomes of Mexican immigrant children: The importance of school context. Child Development, 83, 1477-1485.

Erikson, E.H. (1968). Identity: Youth and crisis. New York, NY: Norton.

García Coll, C.T., Crnic, K., Lamberty, G., Wasik, B.H., Jenkins, R., García, H.V., \& McAdoo, H.P. (1996). An integrative model for the study of developmental competencies in minority children. Child Development, 67, 1891-1914.

Gonzalez, L.M., Stein, G.L., Shannonhouse, L.R., \& Prinstein, M.J. (2012). Latina/o adolescents in an emerging immigrant community: A qualitative exploration of their future goals. Journal of Social Action in Counseling and Psychology, 4, 83-102.

Grzywacz, J.G., Quandt, S.A., Chen, H., Isom, S., Kiang, L., Vallejos, Q., \& Arcury, T.A. (2010). Depressive symptoms among Latino farmworkers across the agricultural season: Individual and contextual influences. Cultural Diversity and Ethnic Minority Psychology, 16, 335-343.

Guarnaccia, P.J., Pincay, I.M., Alegría, M., Shrout, P.E., Lewis-Fernández, R., \& Canino, G.J. (2007). Assessing diversity among Latinos: Results from the NLAAS. Hispanic Journal of Behavioral Sciences, 29, 510-534.

Kiang, L., \& Fuligni, A.J. (2009). Ethnic identity in context: Variations in ethnic exploration and belonging within parent, same-ethnic peer, and different-ethnic peer relationships. Journal of Youth and Adolescence, 38, 732-743.

Kiang, L., Grzywacz, J.G., Marin, A.J., Arcury, T.A., \& Quandt, S.A. (2010). Mental health in immigrants from non-traditional receiving sites. Cultural Diversity and Ethnic Minority Psychology, 16, 386394.

Kiang, L., Harter, S., \& Whitesell, N.R. (2007). Relational expression of ethnic identity in Chinese Americans. Journal of Social and Personal Relationships, 24, 277-296.

Kiang, L., \& Johnson, N. (2013). Adolescents' ethnic self-labeling preferences. Journal of Adolescence, 36 , 815-826.

Kiang, L., \& Luu, J. (2013). Concordance in self and ascribed ethnic labels among Asian American adolescents. Asian American Journal of Psychology, 4, 93-99.

Kiang, L., Perreira, K.M., \& Fuligni, A.J. (2011). Ethnic label use in adolescents from traditional and nontraditional immigrant receiving sites. Journal of Youth and Adolescence, 40, 719-729.

Kiang, L., Supple, A.J., Stein, G., \& Gonzalez, L. (2012). School connectedness and adjustment among Latin American and Asian adolescents in emerging immigrant communities. Journal of Youth and Adolescence, 41, 282-294.

Kiang, L., Witkow, M.R., Baldelomar, O.A., \& Fuligni, A.J. (2010). Change in ethnic identity across the high school years among adolescents with Latin American, Asian, and European backgrounds. Journal of Youth and Adolescence, 39, 683-693.

Lee, R.M., \& Yoo, H.C. (2004). Structure and measurement of ethnic identity for Asian American college students. Journal of Counseling Psychology, 51, 263-269.

Mahalingam, R. (2007). Essentialism, power, and the representation of social categories: A folk sociology perspective. Human Development, 50, 300-319.

Mistry, J., \& Wu, J. (2010). Navigating cultural worlds and negotiating identities: A conceptual model. Human Development, 53, 5-25.

Perreira, K.M., Chapman, M.V., \& Stein, G.L. (2006). Becoming an American parent: Overcoming challenges and finding strength in a new immigrant Latino community. Journal of Family Issues, 27, 1383-1414.

Perreira, K.M., Fuligni, A.J., \& Potochnick, S. (2010). Fitting in: The roles of social acceptance and discrimination in shaping the academic motivations of Latino youth in the U.S. Southeast. Journal of Social Issues, 66, 131-153.

Phinney, J.S. (1992). The Multigroup Ethnic Identity Measure: A new scale for use with diverse groups. Journal of Adolescent Research, 7, 156-176.

Phinney, J.S. (2003). Ethnic identity and acculturation. In K.M. Chun, P.B. Organista, \& G. Marin (Eds.), Acculturation: Advances in theory, measurement, and applied research (pp. 63-81). Washington, DC: American Psychological Association.

Phinney, J.S., \& Ong, A.D. (2007). Conceptualization and measurement of ethnic identity: Current status and future directions. Journal of Counseling Psychology, 54, 271-281.

Roberts, R.E., Phinney, J.S., Masse, L.C., Chen, Y.R., Roberts, C.R., \& Romero, A. (1999). The structure of ethnic identity of young adolescents from diverse ethnocultural groups. The Journal of Early Adolescence, 19, 301-322.

Schwartz, S.J., Syed, M., Yip, T., Knight, G.P., Umana-Taylor, A.J., Rivas-Drake, D., \& Lee, R.M. (2014). Methodological issues in ethnic and racial identity research with ethnic minority populations: Theoretical prediction, measurement issues, and research designs. Child Development, 85, 58-76.

Sellers, R.M., Smith, M.A., Shelton, J.N., Rowley, S.A., \& Chavous, T.M. (1998). Multidimensional model of racial identity: A reconceptualization of African American racial identity. Personality and Social Psychology Review, 2, 18-39. 
Society for Research on Adolescence (SRA) (2010). History \& mission. Retrieved April 25, 2014, from http://www.s-r-a.org/about-sra/history-mission.

- Supple, A.J., Graves, K., Daniel, S., Kiang, L., Su, J., \& Cavanaugh, A. (2013). Ethnic, gender, and age differences in adolescent nonfatal suicidal behaviors. Death Studies, 37, 830-847.

Tseng, V., \& Kiang, L. (2014). Placing Asian American child development within historical context: Implications for research, practice, and policy. Paper presented at the biennial meeting of the Society for Research on Adolescence, Austin, TX.

-Umana-Taylor, A.J., Quintana, S.M., Lee, R.M., Cross, W.E. Jr., Rivas-Drake, D., Schwartz, S.J., Syed, M., Yip, T., \& Seaton, E.; Ethnic and Racial Identity in the 21st Century Study Group (2014). Ethnic and racial identity during adolescence and into young adulthood: An integrated conceptualization. Child Development, 85, 21-39.

Williams, J.L., Tolan, P.H., Durkee, M.I., Francois, A.G., \& Anderson, R.E. (2012). Integrating racial and ethnic identity research into developmental understanding of adolescents. Child Development Perspectives, 6, 304-311.

Yip, T. (2005). Sources of situational variation in ethnic identity and psychological well-being: A palm pilot study of Chinese American students. Personality and Social Psychology Bulletin, 31, 1603-1616.

Yip, T. (2014). Ethnic identity in everyday life: The influence of identity development status. Child Development, 85, 205-219.

Yip, T., \& Fuligni, A.J. (2002). Daily variation in ethnic identity, ethnic behaviors, and psychological wellbeing among American adolescents of Chinese descent. Child Development, 73, 1557-1572.

Yoshikawa, H., Mistry, R.S., \& Wang, Y. (2014). Methodological considerations for research with Asian American youth and families. Paper presented at the biennial meeting of the Society for Research on Adolescence, Austin, TX. 